\title{
PERIODIC SOLUTIONS OF HYPERBOLIC PARTIAL DIFFERENTIAL EQUATIONS IN A STRIP
}

\author{
BY \\ A. K. AZIZ( $\left.{ }^{1}\right)$ AND A. M. MEYERS
}

1. Introduction. In this paper we shall investigate the questions of existence, uniqueness, and continuous dependence on initial data of periodic solutions of the nonlinear hyperbolic equation:

$$
L u \equiv u_{x y}+a(x, y) u_{x}+b(x, y) u_{y}+c(x, y) u=f\left(x, y, u, u_{x}, u_{y}\right)
$$

in the strip $S=\{(x, y) ;-\infty<x<\infty, 0 \leqq y \leqq r\}$. Here the functions $a, b, c$ and $f$ are assumed to be periodic in $x$ with the same period $T$. (For a precise statement of the smoothness assumptions of the functions involved see the statements of the theorems in §3.) We ask for a classical solution of (1.1), periodic in $x$ with period $T$ and satisfying the initial condition

$$
u(x, 0)=\theta(x)
$$

where $\theta(x)$ is continuously differentiable and periodic with period $T$.

The problem mentioned above has been discussed by L. Cesari [2], [3], for the more difficult case where no damping is present, i.e. for the equation

$$
u_{x y}=f\left(x, y, u, u_{x}, u_{y}\right)
$$

Similar problems concerning the existence of periodic solutions for the equation

$$
u_{t t}-u_{x x}=\varepsilon f\left(t, x, u, u_{t}, u_{x}\right)
$$

have been studied by O. Vejvoda [9], J. K. Hale [5] and P. H. Rabinowitz [8]. L. Cesari [4] and J. K. Hale [5] have also considered the question of existence of periodic solutions in the large for (1.3). The approach in [2], [3], [4] and [5] is based on the method used by these authors for similar problems in ordinary differential equations [6]. The approach and techniques used by Rabinowitz can be considered as an extension of the methods of the theory of elliptic boundary value problems to hyperbolic equations. The results presented in the present paper neither contain the results of the previous authors nor are they contained in their

Received by the editors December 10, 1968 and, in revised form, May 15, 1969.

(1) Research of this author was supported in part by the N.S.F. grant GP-8906 and in part by the U. S. Naval Ordnance Laboratory, White Oak, Maryland.

Copyright (O 1969, American Mathematical Society 
results. Due to the presence of damping, we obtain the existence of a solution under weaker hypotheses, e.g., the Lipschitz condition on $f$ is replaced by a more general condition:

$$
|f(x, y, u, p, q)-f(x, y, u, \bar{p}, \bar{q})| \leqq \bar{\omega}_{1}(|p-\bar{p}|)+\bar{\omega}_{2}(|q-\bar{q}|),
$$

where $\bar{\omega}_{1}$ and $\bar{\omega}_{2}$ satisfy certain integral relations (see Hypothesis 4 of Theorem 3.1). In case $\bar{\omega}_{1}$ and $\bar{\omega}_{2}$ are linear in their arguments we have the Lipschitz condition. It appears that the introduction of condition (1.5) simplifies the proofs and makes the results more explicit. Our approach is completely different from those used by the authors cited above. It is based on a representation formula for the general solutions of the hyperbolic equation

$$
u_{x y}+a(x, y) u_{x}+b(x, y) u_{y}+C(x, y, u)=0
$$

due to M. Picone [7]. From an extensive search of the literature, it appears that this procedure has not been fully explored and in particular it has not been used in connection with the periodic solutions of hyperbolic equations. [In the present paper we have confined our discussion to the study of periodic solutions in the strip. The extension of these results to the case of periodic solutions in the large, i.e. the case where we require $u(x+T, y)=u(x, y)=u(x, y+T)$ shall be dealt with in a forthcoming paper.]

In $\$ 2$ we describe Picone's representation formula alluded to earlier and introduce the notations and definitions needed in the sequel.

$\$ 3$ contains the main results concerning the existence, uniqueness and continuous dependence on initial data of the solution of our problem.

2. Preliminaries. In this section we introduce the function spaces and operators which will be used in the sequel to obtain our main results.

Let us suppose for now that $f$ is defined in $S \times E$ ( $E$ is the set of real numbers) and is continuous there. Also suppose $a, a_{x}, b$ and $c$ are continuous in $S$. Picone [7] has shown that it is possible to find functions $\alpha, \beta$ and $\gamma$ such that on a rectangle $\left[0, r_{1}\right] \times\left(0, r_{2}\right]$ (contained in the domain $S$ )

$$
(L u)(x, y)-f(x, y, u) \equiv\left[(1 / \alpha \beta)\left(\alpha(\beta u)_{y}\right)_{x}\right](x, y)-\gamma(x, y, u),
$$

where

$$
L u \equiv u_{x y}+a u_{x}+b u_{y}+c u .
$$

A necessary and sufficient condition for (2.1) to hold is

$$
\text { (i) } \beta_{y} / \beta=a ; \quad \text { (ii) }(\alpha \beta)_{x} / \alpha \beta=b \text {; }
$$

$$
\text { (iii) } \gamma(x, y, u)=f(x, y, u)+\left[a_{x}+a b-c\right] u \text {. }
$$

Now, if $u$ is a function such that

$$
\left(\alpha(\beta u)_{y}\right)_{x}(x, y)=A(x, y, u),
$$


where

$$
A(x, y, u)=(\alpha \beta)(x, y) \gamma(x, y, u)
$$

then

$$
(L u)(x, y)=f(x, y, u)
$$

and conversely. But if $u$ satisfies (2.4) then

$$
u(x, y)=\frac{1}{\beta(x, y)}\left\{\psi(x)+\int_{0}^{y} \frac{1}{\alpha(x, \eta)}\left[\phi(\eta)+\int_{0}^{x} A(\xi, \eta, u) d \xi\right] d \eta\right\}
$$

for arbitrary functions $\psi(x)$ and $\phi(y)$.

Conversely, any function which satisfies (2.5) will satisfy also (2.4) and hence

$$
(L u)(x, y)=f(x, y, u) \text {. }
$$

It is clear that Picone's method may be applied if $f$ depends on $u_{x}$ and $u_{y}$ also. Then one obtains an integral equation similar to (2.5), with $A$ depending on $u_{x}$ and $u_{y}$. We suppose for now that $f$ is defined on $S \times E^{3}$ and we proceed to show how (2.5) is used to answer the question of existence of periodic solutions of $L u=f$.

Assume that $a, b, c, \theta$ and $f$ are continuous in their domains and periodic in $x$ with the same period $T . a_{x}$ and $\theta^{\prime}(x)$ are continuous. Let $R=[0, T] \times[0, r]$. Then by virtue of periodicity of $a, b, c$ and $f$ we need only find a function $u$ such that

$$
\begin{aligned}
L u(x, y) & =f\left(x, y, u, u_{x}, u_{y}\right), \\
u(x, 0) & =\theta(x), \quad u(0, y)=u(T, y), \quad u_{x}(0, y)=u_{x}(T, y) .
\end{aligned}
$$

If such $u$ exists then it must satisfy

$$
u(x, y)=\frac{1}{\beta(x, y)}\left\{\psi(x)+\int_{0}^{y} \frac{1}{\alpha(x, \eta)}\left[\phi(\eta)+\int_{0}^{x} A\left(\xi, \eta, u, u_{x}, u_{y}\right) d \xi\right] d \eta\right\}
$$

along with the conditions

$$
u(x, 0)=\theta(x), \quad u(0, y)=u(T, y), \quad u_{x}(0, y)=u_{x}(T, y) .
$$

Thus we find that

$$
\psi(x)=\theta(x) \beta(x, 0), \quad \phi(y)=\frac{(\alpha \beta)(0, y)}{(\alpha \beta)(T, y)-(\alpha \beta)(0, y)} \int_{0}^{T} A\left(\xi, y, u, u_{x}, u_{y}\right) d \xi .
$$

$\phi$ is well defined provided that

$$
\int_{0}^{T} b(s, y) d s \neq 0 \text { for } y \in[0, r] .
$$

Finally, from the definitions of $\alpha, \beta$ and $A$, if $u$ satisfies (2.6) and if (2.9) holds, then $u$ must satisfy

$$
u(x, y)=\theta(x) \exp \left(-\int_{0}^{y} a(x, t) d t\right)+\int_{0}^{y} \int_{0}^{T} \gamma\left(\xi, \eta, u, u_{x}, u_{y}\right) \frac{s(\xi, \eta ; x, y)}{t(\eta)} d \xi d \eta
$$

$$
+\int_{0}^{y} \int_{0}^{x} \gamma\left(\xi, \eta, u, u_{x}, u_{y}\right) s(\xi, \eta ; x, y) d \xi d \eta
$$


where

$$
\begin{aligned}
\gamma\left(x, y, u, u_{x}, u_{y}\right) & =f\left(x, y, u, u_{x}, u_{y}\right)+\left[\left(a_{x}+a b-c\right) u\right](x, y) \\
s(\xi, \eta ; x, y) & =\exp \left(-\int_{\eta}^{y} a(x, t) d t-\int_{\xi}^{x} b(s, \eta) d s\right) \\
t(\eta) & =\exp \left(\int_{0}^{T} b(s, \eta) d s\right)-1
\end{aligned}
$$

Conversely, a solution $u$ of the integral equation (2.10) will satisfy (2.6).

The equation (2.10) leads us to consider the integral operator

$$
\begin{aligned}
(K u)(x, y)= & \theta(x) \exp \left(-\int_{0}^{y} a(x, t) d t\right) \\
& +\int_{0}^{y} \int_{0}^{T} \gamma\left(\xi, \eta, u, u_{x}, u_{y}\right) \frac{s(\xi, \eta ; x, y)}{t(\eta)} d \xi d \eta \\
& +\int_{0}^{y} \int_{0}^{x} \gamma\left(\xi, \eta, u, u_{x}, u_{y}\right) s(\xi, \eta ; x, y) d \xi d \eta
\end{aligned}
$$

with $(K u)_{x}$ and $(K u)_{y}$ given by

$$
\begin{aligned}
(K u)_{x}(x, y)= & {\left[\theta^{\prime}(x)-\theta(x) \int_{0}^{y} a_{x}(x, t) d t\right] \exp \left(-\int_{0}^{y} a(x, t) d t\right) } \\
& +\int_{0}^{y} \int_{0}^{T} \gamma\left(\xi, \eta, u, u_{x}, u_{y}\right) \frac{s_{x}(\xi, \eta ; x, y)}{t(\eta)} d \xi d \eta \\
& +\int_{0}^{y} \gamma\left(x, \eta, u, u_{x}, u_{y}\right) \exp \left(-\int_{\eta}^{y} a(x, t) d t\right) d \eta \\
& +\int_{0}^{y} \int_{0}^{x} \gamma\left(\xi, \eta, u, u_{x}, u_{y}\right) s_{x}(\xi, \eta ; x, y) d \xi d \eta \\
(K u)_{y}(x, y)= & -a(x, y)(K u)(x, y) \\
& +\frac{1}{t(y)} \int_{0}^{T} \gamma\left(\xi, y, u, u_{x}, u_{y}\right) \exp \left(-\int_{\xi}^{x} b(s, y) d s\right) d \xi \\
& +\int_{0}^{x} \gamma\left(\xi, y, u, u_{x}, u_{y}\right) \exp \left(-\int_{\xi}^{x} b(s, y) d s\right) d \xi
\end{aligned}
$$

The operator $K$ is defined on the space $C_{p}$ of all continuous functions $u$ on $R$, such that $u_{x}$ and $u_{y}$ are continuous on $R$ and $u(0, y)=u(T, y), u_{x}(0, y)=u_{x}(T, y)$. The norm in $C_{p}$ is defined by

where for $\lambda>0$

$$
\|u\|=\|u\|_{\lambda}+\left\|u_{x}\right\|_{\lambda}+\left\|u_{y}\right\|_{\lambda}
$$

$$
\|u\|_{\lambda}=\max _{(x, y) \in R}\left[e^{-\lambda y}|u(x, y)|\right] .
$$

Using the properties of $C_{p}$, the periodicity of the functions $\theta(x), a(x, y), b(x, y)$, $c(x, y)$ and $f\left(x, y, u, u_{x}, u_{y}\right)$ it easily follows from (2.11), (2.12) and (2.13) that $K$ maps $C_{p}$ into itself. 
3. Existence, uniqueness and continuous dependence on initial data. We begin the presentation of our main results by stating four basic hypotheses which we will assume in every theorem. These assumptions are:

$\mathrm{H}_{1}: f$ is continuous on its domain $\left(S \times E^{3}\right)$ and it is periodic in $x$ with period $T$;

$\mathrm{H}_{2}: a, a_{x}, b$ and $c$ are continuous in a strip $S=\{(x, y) ;-\infty<x<\infty, 0 \leqq y \leqq r\}$ and $a, b$ and $c$ are periodic in $x$ with period $T$;

$\mathrm{H}_{3}: \theta(x)$ is continuous with continuous derivative $\theta^{\prime}(x)$ on the real line and $\theta$ is periodic with period $T$;

$\mathrm{H}_{4}: b(x, y)>0$ in $R=[0, T] \times[0, r]$.

We remark that $\mathrm{H}_{4}$ is assumed for technical convenience. The methods of proof are the same for $b(x, y)<0$.

In order to state precisely the conditions under which we show existence it is convenient to develop first some properties of $K$ on a set

$$
A_{\rho}=\left\{u \in C_{p} ;\|u\| \leqq \rho, \rho>0\right\} .
$$

Suppose $f$ is uniformly bounded on $S \times E^{3}$ and let

$$
\begin{aligned}
M & =\sup _{\text {c } S \times E^{3}}|f(x, y, z, p, q)|, & M^{\prime} & =\max \left\{\max _{0 \leqq x \leqq T}|\theta(x)|, \max _{0 \leqq x \leqq T}\left|\theta^{\prime}(x)\right|\right\}, \\
N & =\max _{(x, y) \in R}\left|\left(a_{x}+a b-c\right)(x, y)\right|, & N^{\prime} & =\max \left\{\max _{(x, y) \in R}|a(x, y)|, \max _{(x, y) \in R}\left|a_{x}(x, y)\right|\right\}, \\
m & =\min _{(x, y) \in R} b(x, y), & m^{\prime} & =\max _{(x, y) \in R} b(x, y) .
\end{aligned}
$$

We note that

$$
\begin{aligned}
& |s(\xi, \eta ; x \quad y)| \leqq e^{N^{\prime} r} e^{m(\xi-x)} \quad \text { if } 0 \leqq \xi \leqq x, 0 \leqq \eta \leqq y, \\
& \leqq e^{N^{\prime} r} e^{m^{\prime}(\xi-x)} \text { if } x \leqq \xi \leqq T, 0 \leqq \eta \leqq y .
\end{aligned}
$$

Using the above inequality we find that for $u \in A_{\rho}$

where

$$
\|K u\| \leqq\left(\mu_{1}+\frac{\mu_{2}}{\lambda}\right) \rho+\mu_{3},
$$

$$
\begin{aligned}
\mu & =\frac{1}{m}+\frac{1}{m^{\prime}}\left(\frac{e^{m^{\prime} T}-1}{e^{m T}-1}\right), \\
\mu_{1} & =N \mu, \\
\mu_{2} & =\left[N \mu\left(1+N^{\prime} r+m^{\prime}+N^{\prime}\right)+N\right] e^{N^{\prime} r}, \\
\mu_{3} & =\left[M r \mu\left(1+N^{\prime} r+m^{\prime}+N^{\prime}\right)+M^{\prime}\left(2+N^{\prime} r+N^{\prime}\right)+M r\right] e^{N^{\prime} r}+M \mu .
\end{aligned}
$$

If we require that $N \mu<1$ and choose $\lambda>\mu_{2} /\left(1-\mu_{1}\right)$, then

$$
\rho \geqq \frac{\mu_{3}}{1-\mu_{1}-\mu_{2} / \lambda}
$$

has the property that $K A_{\rho} \subseteq A_{\rho}$. (Note that for $b=$ constant $>2 N$ the inequality $N \mu<1$ is satisfied for all $T>0$; also for $a_{x}+a b-c=0$, i.e. $N=0$, the above inequality is satisfied for all $T>0$.) 
The set $A_{\rho}$ is closed and convex and $K$ is continuous on $A_{\rho}$. We would like to find a closed subset $A$ of $A_{\rho}$ such that $K A \subseteq A$ and $(\overline{K A})$ is compact. To this end we observe that the functions $s(x, y ; \xi, \eta), s_{x}(\xi, \eta ; x, y), s_{y}(\xi, \eta ; x, y)$, $\exp \left(-\int_{\xi}^{x} b(s, y) d s\right) / t(y), \exp \left(-\int_{\xi}^{x} b(s, y) d s\right)$ and $\exp \left(-\int_{\eta}^{y} a(x, t) d t\right)$ are all continuous on the compact set $R \times R$ and thus they possess a common modulus of continuity $\nu_{1}$ on $R \times R$. Similarly $\theta, \theta^{\prime}, a_{x}, a$ and $\left(a_{x}+a b-c\right)$ are all continuous on the closed, bounded rectangle $R$ and have a common modulus of continuity $\nu_{2}$ on $R$. Also for $\rho$ and $\lambda$ such that $K A_{\rho} \subseteq A_{\rho}$ let $\nu_{3}$ denote the modulus of continuity of $f$ on $R \times\left[-\rho e^{\lambda r}, \rho e^{\lambda r}\right]^{3}$. Now, on $[0, \infty)$ let $\eta_{i}(i=1,2,3,4,5,6)$ be defined by

$$
\begin{aligned}
& \eta_{1}(\delta)=\nu_{2}(\delta) e^{N^{\prime} r}+\left[M^{\prime}+\operatorname{Tr}\left(M+N \rho e^{\lambda r}\right) \frac{e^{m T}}{e^{m T}-1}\right] \nu_{1}(\delta) \\
& +r\left(M+N \rho e^{\lambda r}\right) e^{N^{\prime} r+m T} \delta, \\
& \eta_{2}(\delta)=\left[M^{\prime}+\operatorname{Tr}\left(M r+N \rho e^{\lambda r}\right) \frac{e^{m T}}{e^{m T}-1}\right] \nu_{1}(\delta) \\
& +T\left(M+N \rho e^{\lambda r}\right) e^{N^{\prime} r+m T} \frac{e^{m T}}{e^{m T}-1} \delta, \\
& \eta_{3}(\delta)=\left[M^{\prime}+N^{\prime} r+\left(M+N \rho e^{\lambda r}\right)\left(\operatorname{Tr} \frac{e^{m T}}{e^{m T}-1}+r\right)\right] \nu_{1}(\delta) \\
& +\left[M^{\prime} N^{\prime} e^{N^{\prime} r}+\left(M+N \rho e^{\lambda r}\right)\left(T\left(N^{\prime} r+m^{\prime}\right) e^{N^{\prime} r+m^{\prime} T} \frac{e^{m T}}{e^{m T}-1}+e^{N^{\prime} r}\right)\right] \delta, \\
& \eta_{4}(\delta)=N^{\prime} \eta_{1}(\delta)+T\left(M+N \rho e^{\lambda r}\right) \frac{e^{m T}}{e^{m T}-1} \nu_{1}(\delta)+\rho e^{\lambda r} \nu_{2}(\delta) \\
& +\left(M+N \rho e^{\lambda r}\right) e^{m^{\prime} T} \delta \\
& \eta_{5}(\delta)=\left[M^{\prime}+N^{\prime} M^{\prime} r+\left(M+N \rho e^{\lambda r}\right)\left(\operatorname{Tr} \frac{e^{m T}}{e^{m T}-1}+r\right)\right] \nu_{1}(\delta) \\
& +\left[1+M^{\prime} r+N^{\prime} r+\rho r e^{\lambda r}\right] e^{N^{\prime} r} \nu_{2}(\delta)+e^{N^{\prime} r} r \nu_{3}(\delta) \\
& +\left(M+N \rho e^{\lambda r}\right)\left(N^{\prime} r+m^{\prime}\right) r e^{N^{\prime} r+m^{\prime} T} \delta \text {, } \\
& \eta_{6}(\delta)=N^{\prime} \eta_{2}(\delta)+2 T\left(M+N \rho e^{\lambda r}\right) \nu_{1}(\delta)+\left(\rho e^{\lambda r}+T \rho e^{\lambda r} e^{m^{\prime} T} \frac{e^{m T}}{e^{m T}-1}\right) \nu_{2}(\delta) \\
& +T e^{m^{\prime} T} \frac{e^{m T}}{e^{m T}-1} \nu_{3}(\delta) \text {. }
\end{aligned}
$$

We observe that the functions $\nu_{i}$ (and hence $\eta_{i}$ ) are continuous, nonnegative, nondecreasing and vanish at the origin. Without loss of generality we may assume that $\nu_{i}$ are subadditive.

Finally we set

$$
\begin{aligned}
& C_{0}=\max _{0 \leqq \eta \leqq y ;(x, y) \in R} \exp \left(-\int_{\eta}^{y} a(x, t) d t\right), \\
& C_{1}=\max _{0 \leqq \xi \leqq x ;(x, y) \in R} \exp \left(-\int_{\xi}^{x} b(s, y) d s\right), \\
& C_{2}=\max _{0 \leqq \xi \leqq T ;(x, y) \in R} \frac{\exp \left(-\int_{\xi}^{x} b(s, y) d s\right)}{t(y)} .
\end{aligned}
$$


Then for $u \in A_{\rho}$ and $(x, y)$ and $(\bar{x}, \bar{y})$ in $R$ one may verify that

$$
\begin{aligned}
& |K u(x, y)-K u(\bar{x}, y)| \leqq \eta_{1}(|x-\bar{x}|), \\
& |K u(x, y)-K u(x, \bar{y})| \leqq \eta_{2}(|y-\bar{y}|), \\
& \left|(K u)_{x}(x, y)-(K u)_{x}(x, \bar{y})\right| \leqq \eta_{3}(|y-\bar{y}|) \text {, } \\
& \left|(K u)_{y}(x, y)-(K u)_{y}(\bar{x}, y)\right| \leqq \eta_{4}(|x-\bar{x}|) \text {, } \\
& \left|(K u)_{x}(x, y)-(K u)_{x}(\bar{x}, y)\right| \leqq \eta_{5}(|x-\bar{x}|)+N e^{N^{\prime} r} \int_{0}^{y}|u(x, \eta)-u(\bar{x}, \eta)| d \eta \\
& +e^{N^{\prime} r} \int_{0}^{y} \nu_{3}(|u(x, \eta)-u(\bar{x}, \eta)|) d \eta \\
& +C_{0} \int_{0}^{y} \mid f\left(x, \eta, u(x, \eta), u_{x}(x, \eta), u_{y}(x, \eta)\right) \\
& -f\left(x, \eta, u(x, \eta), u_{x}(\bar{x}, \eta), u_{y}(\bar{x}, \eta)\right) \mid d \eta \\
& \left|(K u)_{y}(x, y)-(K u)_{y}(x, \bar{y})\right| \leqq \eta_{8}(|y-\bar{y}|)+\frac{N e^{m T}}{e^{m T}-1} \int_{0}^{T}|u(\xi, y)-u(\xi, \bar{y})| d \xi \\
& +N e^{m r} \int_{0}^{x}|u(\xi, y)-u(\xi, \bar{y})| d \xi \\
& +\frac{e^{m T}}{e^{m T}-1} \int_{0}^{T} \nu_{3}(|u(\xi, y)-u(\xi, \bar{y})|) d \xi \\
& +e^{m T} \int_{0}^{x} \nu_{3}(|u(\xi, y)-u(\xi, \bar{y})|) d \xi \\
& +C_{2} \int_{0}^{T} \mid f\left(\xi, \bar{y}, u(\xi, \bar{y}), u_{x}(\xi, y), u_{y}(\xi, y)\right) \\
& -f\left(\xi, \bar{y}, u(\xi, \bar{y}), u_{x}(\xi, \bar{y}), u_{y}(\xi, \bar{y})\right) \mid d \xi \\
& +C_{1} \int_{0}^{x} \mid f\left(\xi, \bar{y}, u(\xi, \bar{y}), u_{x}(\xi, y), u_{y}(\xi, y)\right) \\
& -f\left(\xi, \bar{y}, u(\xi, \bar{y}), u_{x}(\xi, \bar{y}), u_{y}(\xi, \bar{y})\right) \mid d \xi .
\end{aligned}
$$

Now we can state and prove our first result:

THEOREM 3.1. Suppose

(1) Hypotheses $\mathrm{H}_{1}-\mathrm{H}_{4}$ hold;

(2) $a, b, c$ and $T$ are such that $N\left[1 / m+\left(1 / m^{\prime}\right)\left(\left(e^{m^{\prime} T}-1\right) /\left(e^{m T}-1\right)\right)\right]<1$;

(3) $f$ is uniformly bounded in $S \times E^{3}$;

(4) $f$ satisfies in $S \times E^{3}$

$$
|f(x, y, z, p, q)-f(x, y, z, \bar{p}, \bar{q})| \leqq \bar{\omega}_{1}(|p-\bar{p}|)+\bar{\omega}_{2}(|q-\bar{q}|),
$$

where $\bar{\omega}_{1}$ and $\bar{\omega}_{2}$ are nonnegative, nondecreasing, subadditive, continuous functions which vanish at the origin, and further $\bar{\omega}_{1}$ and $\bar{\omega}_{2}$ are such that for

$$
\begin{aligned}
& \Omega_{1}(\delta)=\eta_{5}(\delta)+\left[N \eta_{1}(\delta)+\nu_{3}\left(\eta_{1}(\delta)\right)+\bar{\omega}_{2}\left(\eta_{4}(\delta)\right)\right] r e^{N^{\prime} r} \\
& \Omega_{2}(\delta)=\eta_{8}(\delta)+e^{m^{\prime} T} \frac{e^{m T}}{e^{m T}-1}\left[T N \eta_{2}(\delta)+T \nu_{3}\left(\eta_{2}(\delta)\right)+T \bar{\omega}_{1}\left(\eta_{3}(\delta)\right)\right]
\end{aligned}
$$


the solutions $\rho_{1}(t, \delta)$ and $\rho_{2}(t, \delta)$ of the equations

$$
\begin{aligned}
& \rho_{1}(t, \delta)=\Omega_{1}(\delta)+C_{0} \int_{0}^{t} \bar{\omega}_{1}\left(\rho_{1}(\tau, \delta)\right) d \tau, \quad t \geqq 0, \\
& \rho_{2}(t, \delta)=\Omega_{2}(\delta)+C_{1} \int_{0}^{t} \bar{\omega}_{2}\left(\rho_{2}(\tau, \delta)\right) d \tau+C_{2} \int_{0}^{T} \bar{\omega}_{2}\left(\rho_{2}(\tau, \delta)\right) d \tau, \quad t \in[0, T],
\end{aligned}
$$

are such that $\lim _{0 \rightarrow 0} \rho_{i}(t, \delta)=0(i=1,2)$ uniformly in $t$.

Then there exists a function $u$ in $S$ such that

$$
(L u)(x, y)=f\left(x, y, u, u_{x}, u_{y}\right), \quad u(x, 0)=\theta(x), \quad u(x+T, y)=u(x, y) .
$$

Proof. According to the discussion preceding the statement of the theorem, the second hypothesis assures the existence of a number $\rho>0$ such that $K A_{\rho} \subseteq A_{\rho}$. Further, if we let $A$ be the set of all $u \in A_{\rho}$ such that

$$
\begin{aligned}
&|u(x, y)-u(\bar{x}, y)| \leqq \eta_{1}(|x-\bar{x}|), \\
&|u(x, y)-u(x, \bar{y})| \leqq \eta_{2}(|y-\bar{y}|), \\
&\left|u_{x}(x, y)-u_{x}(x, \bar{y})\right| \leqq \eta_{3}(|y-\bar{y}|), \\
&\left|u_{y}(x, y)-u_{y}(\bar{x}, y)\right| \leqq \eta_{4}(|x-\bar{x}|), \\
&\left|u_{x}(x, y)-u_{x}(\bar{x}, y)\right| \leqq \Omega_{1}(|x-\bar{x}|)+C_{0} \int_{0}^{y} \bar{\omega}_{1}\left(\rho_{1}(t,|x-\bar{x}|)\right) d t, \\
&\left|u_{y}(x, y)-u_{y}(x, \bar{y})\right| \leqq \Omega_{2}(|y-\bar{y}|)+C_{1} \int_{0}^{x} \bar{\omega}_{2}\left(\rho_{2}(t,|y-\bar{y}|)\right) d t \\
&+C_{2} \int_{0}^{T} \bar{\omega}_{2}\left(\rho_{2}(t,|y-\bar{y}|)\right) d t,
\end{aligned}
$$

then (4) implies that $K A \subseteq A$ and $A$ is compact. $A$ is also closed and convex and hence by Schauder's theorem there is at least one $u \in A$ such that $K u=u$. The function $u$ whose existence is asserted by the theorem is obtained by extending $u$ periodically to all of $S$.

In the special case of linear functions $\bar{\omega}_{i}$, i.e. when $f$ satisfies a Lipschitz condition in the last two variables we have

\section{Corollary 3.1. Suppose}

(1) The first three conditions of Theorem 3 hold;

(2) $|f(x, y, z, p, q)-f(x, y, z, \bar{p}, \bar{q})| \leqq L_{1}|p-\bar{p}|+L_{2}|q-\bar{q}|$;

(3) $\exp \left(C_{1} L_{2} T\right)<1+C_{1} / C_{2}$.

Then the conclusion of Theorem 3.1 follows.

Proof. We need only to show that the hypotheses of the corollary imply the fourth condition of the theorem. Let

$$
r_{1}(t, \delta)=\int_{0}^{t} \rho_{1}(\tau, \delta) d \tau, \quad t \geqq 0, \quad r_{2}(t, \delta)=\int_{0}^{t} \rho_{2}(\tau, \delta) d \tau, \quad t \in[0, T] .
$$


Then the integral equations in the fourth hypothesis of Theorem 3.1 are equivalent to the differential equations

(i) $r_{1}^{\prime}(t, \delta)-C_{0} L_{1} r_{1}(t, \delta)=\Omega_{1}(\delta), r_{1}(0, \delta)=0$,

(ii) $r_{2}^{\prime}(t, \delta)-C_{1} L_{2} r_{2}(t, \delta)=\Omega_{2}(\delta)+C_{2} L_{2} r_{2}(T, \delta), r_{2}(0, \delta)=0$,

which have the solutions

$$
\begin{aligned}
& r_{1}(t, \delta)=\Omega_{1}(\delta) \frac{\exp \left(C_{0} L_{1} t\right)-1}{C_{0} L_{1}} \\
& r_{2}(t, \delta)=\Omega_{2}(\delta) \exp \left(C_{1} L_{2} t\right)-1 \frac{1}{C_{1} L_{2}-C_{2} L_{2}\left(\exp \left(C_{1} L_{2} T\right)-1\right)} .
\end{aligned}
$$

Thus $\lim _{\delta \rightarrow 0} r_{i}(t, \delta)=0(i=1,2)$ uniformly in $t$ since $\Omega_{1}$ and $\Omega_{2}$ are continuous and vanish at zero. Further, $\rho_{t}(t, \delta) \geqq 0(i=1,2)$ so that $\lim _{\delta \rightarrow 0} \rho_{i}(t, \delta)=0$ uniformly in $t$. Hence the hypotheses of Theorem 3.1 are satisfied and the corollary is proved.

REMARK 1 . In case $f$ is not uniformly bounded in $S \times E^{3}$ but is bounded on a set $D=S \times[-d, d]^{3}$, where $\sup _{(x, y, z, p, q) \in D}|f(x, y, z, p, q)| \leqq M_{d}$, and satisfies hypothesis (4) of Theorem 3.1 in $D$ we can show that the conclusion of Theorem 3.1 follows if $d$ is sufficiently large. More precisely, let $M$ be replaced by $M_{d}$ in the expressions (3.1) and require that, besides $\mu_{1}=N \mu<1$, we have $r<\left(1-\mu_{1}\right) / \mu_{2}$, and

$$
d \geqq \mu 3 /\left(1-\mu_{1}-\mu_{2} r\right) \text {. }
$$

Then the set

$$
A_{d}=\left\{u \in C_{p} ;\left(\max _{(x, y) \in R}|u(x, y)|+\max _{(x, y) \in R}\left|u_{x}(x, y)\right|+\max _{(x, y) \in R}\left|u_{y}(x, y)\right|\right) \leqq d\right\}
$$

is mapped into itself by $K$, and using $M_{d}$ instead of $M$ in the remainder of the proof of Theorem 3.1 one can show that the conclusion of this theorem follows.

THEOREM 3.2. Suppose

(1) $\mathrm{H}_{1}-\mathrm{H}_{4}$ hold;

(2) $|f(x, y, z, p, q)-f(x, y, \bar{z}, \bar{p}, \bar{q})| \leqq L\{|z-\bar{z}|+|p-\bar{p}|+|q-\bar{q}|\}$;

(3) $a, b, c$ and $T$ are such that $(L+N)\left[1 / m+\left(1 / m^{\prime}\right)\left(\left(e^{m^{\prime} T}-1\right) /\left(e^{m T}-1\right)\right)\right]<1$.

Then there is a unique function $u$ for which the conclusion of Theorem 3.1 holds.

Proof. The theorem is proved by showing that $K$ is a contraction mapping on $C_{p}$. Let $u$ and $\bar{u}$ be in $C_{p}$. Then with $\mu, N, N^{\prime}, m^{\prime}$ and $m$ as before

$$
\begin{aligned}
&\|K u(x, y)-K \bar{u}(x, y)\|_{\lambda} \leqq e^{N^{\prime} r}(L+N)(\mu / \lambda)\|u-\bar{u}\|, \\
&\left\|(K u)_{x}(x, y)-(K \bar{u})_{x}(x, y)\right\|_{\lambda} \leqq e^{N^{\prime} r}(L+N)\left[\left(N^{\prime} r+m^{\prime}\right) \mu / \lambda+1 / \lambda\right]\|u-\bar{u}\|, \\
&\left\|(K u)_{y}(x, y)-(K \bar{u})_{y}(x, y)\right\|_{\lambda} \leqq\left[e^{v^{\prime} r}(L+N) N^{\prime} \mu / \lambda+(L+N) \mu\right]\|u-\bar{u}\| .
\end{aligned}
$$

Thus

$$
\|K u-K \bar{u}\| \leqq\left\{\left(\left[\mu+\left(N^{\prime} r+m^{\prime}\right) \mu+N^{\prime} \mu+1\right] / \lambda\right)(L+N) e^{N^{\prime} r}+(L+N) \mu\right\}\|u-\bar{u}\| .
$$


Now by the third condition of the theorem, $(L+N) \mu<1$ so that by choosing

$$
\lambda>\left(\mu+\left(N^{\prime} r+m^{\prime}\right) \mu+N^{\prime} \mu+1\right)(L+N) e^{N^{\prime} r} /[1-(L+N) \mu]
$$

$K$ will be a contraction mapping on $C_{p}$. Thus the theorem is proved.

REMARK 2. Again, the global Lipschitz condition of Theorem 3.2 may be relaxed. Suppose there is a number $d$ such that the Lipschitz condition of hypothesis (2) holds in $D$ (see Remark 1) with Lipschitz constant $L_{d}$ and hypothesis (3) is satisfied for $L_{d}$. Then we find that $K$ maps $A_{d}$ (see Remark 1) into itself provided $\mu_{1}<1$, $r<\left(1-\mu_{2}\right) / \mu_{2}, d \geqq \mu_{3} /\left(1-\mu_{1}-\mu_{2} r\right)$ where now

$$
\begin{aligned}
\mu_{1} & =\left(L_{d}+N\right) \mu, \\
\mu_{2} & =\left(L_{d}+N\right)\left[1+\left(1+N^{\prime} r+m^{\prime}+N^{\prime}\right) \mu\right] e^{N^{\prime} r}, \\
\mu_{3} & =\left[M^{\prime}\left(2+N^{\prime} r+N^{\prime}\right)+M_{0} r\left\{\left(1+N^{\prime} r+m^{\prime}+N^{\prime}\right) \mu+1\right\}\right] e^{N^{\prime} r}+M_{0} \mu, \\
M_{0} & =\max _{(x, y) \in R}|f(x y, 0,0,0)| .
\end{aligned}
$$

Now the estimates (3.3) with $L$ replaced by $L_{d}$ imply that $K$ is a contraction of $A_{d}$. Thus we obtain the result of Theorem 3.2.

Before stating our final theorem we give an example in connection with our last remark.

AN EXAMPle. Let

$$
L u=u_{x y}+2 u_{y}, \quad f(x, y, z, p, q)=10^{-1}\left(z^{2} \sin x+p^{2}+q^{2}\right), \quad \theta(x)=(\cos x) / 4 .
$$

On $S \times[-d, d]^{3}, d>0$, we have

$$
|f(x, y, z, p, q)-f(x, y, \bar{z}, \bar{p}, \bar{q})| \leqq 5^{-1} d(|z-\bar{z}|+|p-\bar{p}|+|q-\bar{q}|) .
$$

If $r$ and $d$ satisfy the inequalities

$$
5 / 2(5-d-4 d r) \leqq d<5, \quad 4 d r<5-d,
$$

then according to Remark 2 , there is a unique function $\dot{u}$ such that

$$
L u=f, \quad u(x, 0)=\theta(x), \quad u(x, y)=u(x+2 \pi, y) .
$$

If $r<3 / 8(3.4)$ is satisfied by choosing

$$
\frac{1}{2(1+4 r)}\left(5-(5(3-8 r))^{1 / 2}\right) \leqq d \leqq \frac{1}{2(1+4 r)}\left(5+(5(3-8 r))^{1 / 2}\right)
$$

since the right-hand inequality certainly implies $4 d r<5-d$.

Finally, we show the continuous dependence of the unique solution $u$, whose existence is asserted by Theorem 3.2, on the initial function $\theta(x)$. For the sake of simplicity of computation we show how this result is obtained for the case $a=c=0$ and $b>0$. 
THEOREM 3.3. Suppose $a=c=0, b=$ constant and the hypotheses of Theorem 3.2 are satisfied. Let $u^{i}(i=1,2)$ be functions on $S$ such that

$$
\begin{aligned}
& \left(L u^{i}\right)(x, y)=f\left(x, y, u^{i}, u_{x}^{i}, u_{y}^{i}\right), \quad u^{i}(x, 0)=\theta^{i}(x), \quad u^{i}(x, y)=u^{i}(x+T, y) . \\
& \text { If } \quad \max _{x \in[0 . T]}\left\{\left|\theta^{1}(x)-\theta^{2}(x)\right|+\left|d \theta^{1}(x) / d x-d \theta^{2}(x) / d x\right|\right\}<\varepsilon, \quad((r+1) / b+2 r) L<1,
\end{aligned}
$$

then there exists a number $\delta$ such that

$$
\max _{(x, y) \in R}\left\{\left|u^{1}(x, y)-u^{2}(x, y)\right|+\left|u_{x}^{1}(x, y)-u_{x}^{2}(x, y)\right|+\left|u_{y}^{1}(x, y)-u_{y}^{2}(x, y)\right|\right\} \leqq \delta \varepsilon .
$$

Proof. Let

$$
W=u^{1}-u^{2}, \quad \delta f=f\left(x, y, W+u^{2}, W_{x}+u_{x}^{2}, W_{y}+u_{y}^{2}\right)-f\left(x, y, u^{2}, u_{x}^{2}, u_{y}^{2}\right) .
$$

$W$ satisfies on $R$

$$
L W=\delta f, \quad W(x, 0)=\theta^{1}(x)-\theta^{2}(x), \quad W(0, y)=W(T, y) .
$$

Now let a sequence of continuous functions $\left\{W^{n}(x, y)\right\}$ be defined on $R$ by

$$
\begin{aligned}
W^{0}(x, y)= & \theta^{1}(x)-\theta^{2}(x) \\
W^{n}(x, y)= & W^{0}(x, y)+\frac{e^{-b x}}{e^{b T}-1} \int_{0}^{y} \int_{0}^{T} \delta f\left(\xi, \eta, W^{n-1}, W_{x}^{n-1}, W_{y}^{n-1}\right) e^{b \xi} d \xi d \eta \\
& +e^{-b x} \int_{0}^{y} \int_{0}^{x} \delta f\left(\xi, \eta, W^{n-1}, W_{x}^{n-1}, W_{y}^{n-1}\right) e^{b \xi} d \xi d \eta, \quad n \geqq 1 .
\end{aligned}
$$

These functions are well defined. Moreover $W_{x}^{n}(x, y)$ and $W_{y}^{n}(x, y)$ exist and are defined by

$$
\begin{aligned}
W_{x}^{0}(x, y)= & d \theta^{1}(x) / d x-d \theta^{2}(x) / d x \\
W_{x}^{n}(x, y)= & W_{x}^{0}(x, y)-\frac{b e^{-b x}}{e^{b T}-1} \int_{0}^{y} \int_{0}^{T} \delta f\left(\xi, \eta, W^{n-1}, W_{x}^{n-1}, W_{y}^{n-1}\right) e^{b \xi} d \xi d \eta \\
& -b e^{-b x} \int_{0}^{y} \int_{0}^{x} \delta f\left(\xi, \eta, W^{n-1}, W_{x}^{n-1}, W_{y}^{n-1}\right) e^{b \xi} d \xi d \eta \\
& +\int_{0}^{y} \delta f\left(x, \eta, W^{n-1}, W_{x}^{n-1}, W_{y}^{n-1}\right) d \eta, \quad n \geqq 1, \\
W_{y}^{0}(x, y)= & 0, \\
W_{y}^{n}(x, y)= & \frac{e^{-b x}}{e^{-b T}-1} \int_{0}^{T} f\left(\xi, y, W^{n-1}, W_{x}^{n-1}, W_{y}^{n-1}\right) e^{b \xi} d \xi \\
& +e^{-b x} \int_{0}^{x} \delta f\left(\xi, y, W^{n-1}, W_{x}^{n-1}, W_{y}^{n-1}\right) e^{b \xi} d \xi, \quad n \geqq 1 .
\end{aligned}
$$

For $n \geqq 1$, it follows by induction that

$$
\begin{aligned}
& \left|W^{n}(x, y)-W^{n-1}(x, y)\right| \leqq \varepsilon L^{n} \alpha^{n-1} r / b \\
& \left|W_{x}^{n}(x, y)-W^{n-1}(x, y)\right| \leqq 2 \varepsilon L^{n} \alpha^{n-1} r \\
& \left|W_{y}^{n}(x, y)-W_{y}^{n-1}(x, y)\right| \leqq \varepsilon \frac{L^{n} \alpha^{n-1}}{b}
\end{aligned}
$$


where $\alpha=((r+1) / b+2 r)$. Thus

$$
\max _{(x, y) \in R}\left\{\left|W^{n}-W^{n-1}\right|+\left|W_{x}^{n}-W_{x}^{n-1}\right|+\left|W_{y}^{n}-W_{y}^{n-1}\right|\right\} \leqq \varepsilon(L \alpha)^{n} .
$$

Now it follows from the hypotheses $L \alpha<1$ that the sequences

$$
\begin{aligned}
& W^{n}(x, y)=W^{0}(x, y)+\sum_{K=0}^{n-1}\left[W^{K+1}(x, y)-W^{K}(x, y)\right], \\
& W_{x}^{n}(x, y)=W_{x}^{0}(x, y)+\sum_{K=0}^{n-1}\left[W_{x}^{K+1}(x, y)-W_{x}^{K}(x, y)\right], \\
& W_{y}^{n}(x, y)=\sum_{K=0}^{n-1}\left[W_{y}^{K+1}(x, y)-W_{y}^{K}(x, y)\right]
\end{aligned}
$$

converge uniformly and absolutely to continuous functions $\bar{W}, \bar{W}_{x}, \bar{W}_{y}$ respectively and $\bar{W}$ satisfies

$$
L \bar{W}=\delta f, \quad \bar{W}(x, 0)=\theta^{1}(x)-\theta^{2}(x), \quad \bar{W}(0, y)=\bar{W}(T, y) .
$$

Thus by uniqueness $\bar{W}=u^{1}-u^{2}$.

Now from (3.5) we have

$$
\max _{(x, y) \in R}\left\{|\bar{W}(x, y)|+\left|\bar{W}_{x}(x, y)\right|+\left|\bar{W}_{y}(x, y)\right|\right\} \leqq \varepsilon\left(1+\sum_{K=0}^{\infty}(L \alpha)^{K}\right)<\frac{2 \varepsilon}{1-L \alpha} .
$$

This completes the proof.

\section{REFERENC̣ES}

1. A. K. Aziz, Periodic solutions of hyperbolic partial differential equations, Proc. Amer. Math. Soc. 17 (1966), 557-566.

2. L. Cesari, a) Periodic solutions of hyperbolic partial differential equations, Internat. Sympos. on Nonlinear Differential Equations and Nonlinear Mechanics (Colorado Springs, 1961), Academic Press, New York, 1963, pp. 33-57; b) Proc. Internat. Sympos. Non-Linear Vibrations, vol. II (Kiev, 1961), Izdat. Akad. Nauk Ukrain. SSR, Kiev, 1963, pp. 440-457.

3. - A criterion for the existence in a strip of periodic solutions of hyperbolic partial differential equations, Rend. Circ. Mat. Palermo (2) 14 (1965), 1-24.

4. - Existence in the large of periodic solutions of hyperbolic partial differential equations, Arch. Rational Mech. Anal. 20 (1965), 170-190.

5. J. K. Hale, Periodic solutions of a class of hyperbolic equations containing a small parameter, Arch. Rational Mech. Anal. 23 (1966), 380-398.

6. - Oscillations in nonlinear systems, McGraw-Hill, New York, 1963.

7. M. Picone, Sulle equazioni alle derivate parziali del second' ordine del tipo iperbolico in due variabili independenti, Rend. Circ. Mat. Palermo 30 (1910), 349-376.

8. P. H. Rabinowitz, Periodic solutions of nonlinear hyperbolic partial differential equations, Comm. Pure Appl. Math. 20 (1967), 145-205.

9. O. Vejvoda, "Nonlinear boundary value problems for differential equations" in Differential equations and their applications, Czech. Acad. Sci., Prague, 1963, pp. 199-215.

UNIVERSITY OF MARYLAND,

BaLtimore, MaRYLAND

U. S. Naval Ordnance Laboratory, WhITE OAK, MARYLAND 